\title{
Prism adaptation with simultaneous receipt of normal input ${ }^{\prime}$
}

\author{
JOAN E. FOLEY2 \\ UNIVERSITY OF TORONTO
}

\begin{abstract}
A 20-diopter vertically-displacing prism was worn on one eye in conjunction with plane glass on the other. Five groups of Ss differed in the density of a neutral filter worn over one eye. No aftereffect could be measured in either eye in any condition where the luminance of the "normal" field was equal to or greater than that of the prism field. As the luminance of the "normal" field is decreased below that of the prism field, the magnitude of aftereffect increases and is measurable in each eye.
\end{abstract}

A human adult who walks about for some minutes wearing wedge prisms in front of his eyes shows an adaptation to the rearrangement of his visual field. One way in which this adaptation can be measured is to have $S$ point to the apparent position of a small target in a homogeneous field both before and after the prisms are worn. If these tests are performed with normal vision, there is a shift in judgment in the direction of the prism base. This aftereffect can be obtained with either binocular or monocular exposure to the prism (e.g., Held, 1965; Hajos \& Ritter, 1965).

Several conditions where the two eyes are subject to different treatments in a prism-adaptation situation were included in a study by Foley and Miyanishi (1969). In one condition, Ss wore a 20-diopter wedge prism, base-up, on the right eye in conjunction with plane glass on the left. No aftereffects were obtained in this condition, whereas Ss treated otherwise identically, but for whom the left eye was occluded, produced measurable aftereffects regardless of which eye was used in testing. It was speculated that the continuation of normal input inhibits the processing of information in the rearranged field. An alternative explanation was in terms of the content of the field of view. The field of the eye with plane glass includes more of the ground than that of the base-up prism, and it might be the case that information from the ground receives more attention during locomotion. Consistent with this explanation was the observation that, in another condition, information from the field of a base-down prism appeared to contribute more to spatial judgments than that from a base-up prism worn concurrently. The view that normalcy might indeed be a special case was encouraged, nevertheless, by another feature of the results. In spite of the overriding influence of the base-down prism, the "up-and-down" condition produced an interocular difference consistent in direction with the difference in treatments of the eyes. This result was replicated by Foley (1970). In contrast, where normal vision was present, no difference was found between the eyes on monocular tests.

The present study aimed to clarify this situation by combining normal vision in one or the other eye with either up or down base orientations of the prism worn contralaterally. These conditions were also extended to the case in which the two eyes are subject to unequal luminance of their fields, through the use of neutral filters of various densities.

\section{Subjects \\ METHOD}

One hundred and sixty university and high-school students participated. Their ages ranged from 17 to 40 years, and approximately half of them were male. They were allocated to treatment groups at random.

\section{Apparatus}

A 20-diopter wedge prism was mounted over one of two apertures in an opaque face in each of two modified diving masks. The prism could be rotated in its mount to any desired base orientation. A piece of plane glass was mounted over the other aperture. The visual field of each eye was circular and measured about $70 \mathrm{deg}$. Inside the masks, brackets were provided to hold neutral density filters over either eye, as desired. Three glass-mounted Kodak Wratten filters, of $\mathrm{ND} .7,1.3$, and 1.9, respectively, were available. These corresponded to transmission factors of .2 , .05 , and .0125 . An opaque cover (DN $\infty$, transmission 0 ) was also available.

The test apparatus comprised a vertical array of dim circular lights, $20 \mathrm{~min}$ in diam and about $58 \mathrm{~cm}$ from the eye of $S$, whose head was positioned by means of a head and chin rest in conjunction with a head strap. Removable shields permitted monocular presentation of the test lights, the virtual image of which appeared to be directly ahead of $S$. The apparent position of the target light, viewed in darkness, was indicated by the $S$, who reached forward with his right hand and made a mark with a felt pen on a sheet of paper. The marking sheet could not be seen by $S$.

\section{Procedure}

Following 12 practice judgments, the $S$ was pretested. Most judgments were of a target (T) at eye level, but "dummies" randomly selected from two higher and two lower targets within 5 deg of the central one were also presented. Dummy targets were presented as a precaution against stereotyped arm movement within or between test sessions. In particular, such stereotyping could obscure interocular differences where right- and left-eye tests are mixed. Twelve trials were given at pretest and at each subsequent test session. A table of random permutations was used on each occasion to intermix four $T$ and two dummy presentations to each eye. Only $\mathrm{T}$ judgments were analyzed.

After pretesting, $S$ was fitted with a pair of goggles appropriate to the treatment to which he had been allocated. He then spent $10 \mathrm{~min}$ in the company of $\mathrm{E}$, and usually with another $S$, walking along corridors in the building. Overhead lights provided fairly even illumination at about $22 \mathrm{fc}$. He was then tested without goggles.

\section{Design}

Thirty-two Ss were allocated to each of the five filter conditions: ND $0, .7,1.3$, 1.9 , and $\infty$. Half the $S s$ in each group wore the filter in conjunction with the prism and half with the plane glass. In each of these subgroups, half wore the prism in the base-up orientation and half base down, and within each of these half wore the prism on the right eye and half on the left. That is, four Ss were run in each of the 40 combinations of filter density, filter arrangement (with respect to prism or glass), base direction, and prism arrangement (with respect to eye). In the case of Ss not wearing a filter (ND 0), the filter arrangement condition has no meaning, but for purposes of analysis individuals were arbitrarily preallocated to subgroups with respect to this variable.

Eye tested (whether it was exposed through prism or glass) was the only within $S$ variable in the experiment.

\section{RESULTS}

Performance was expressed as an angular shift from pre- to posttest performance (on 


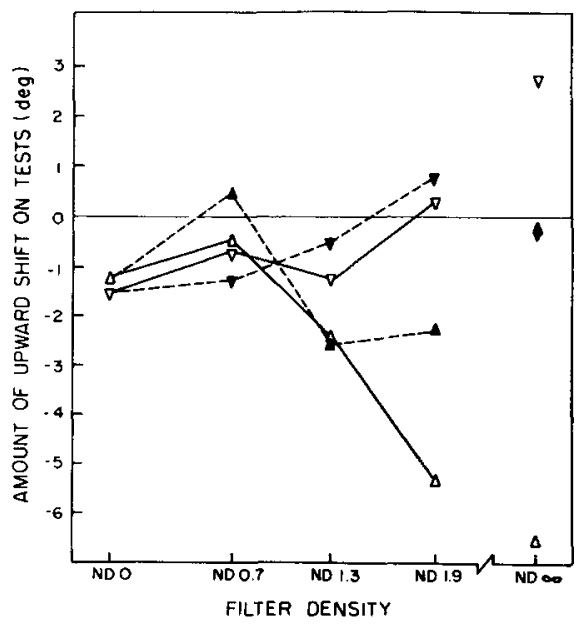

Fig. 1. Magnitude and direction of aftereffect as a function of the density of a neutral filter worn on one eye during binocular exposure. Measures for the two eyes are combined. Base orientation of the prism worn on one eye during exposure is shown by the orientation of triangles on the graph. The filter arrangement during exposure is indicated by the color of the triangle, e.g., $\nabla$ measurement after exposure to base-up prism in conjunction with reduced luminance of the normal field; $\Delta$ measurement after exposure to base-down prism with the luminance of the prism field reduced relative to that of the normal field.

$T$ tests only) for a given eye in each $S$ (aftereffect). An upward (+) shift would be adaptive for the base-up prism and a downward ( - ) shift for the base-down prism. Complete compensation demands a shift of about 11 deg.

Analysis of variance yielded a main effect of base direction which was significant at the .01 level of confidence $[F(1,120)=10.67]$. This difference is in the expected direction, i.e., the mean for all groups with the prism base down-is more negative than that for all groups with the prism base up. However, the Base Direction by Density interaction is also significant at the .01 level $[F(4,120)=3.97]$, reflecting the fact that the base direction effect develops as filter density increases. Further, the Base Direction by Filter Arrangement interaction term reaches the .05 confidence level $[F(1,120)=5.70]$. Inspection of the means for this interaction shows that the base direction effect appears only when the filter is worn on the "normal" field. The higher-order interaction of Base Direction by Density by Filter Arrangement is plotted in Fig. 1. This interaction term falls just short of the .05 level of confidence $[F(4,120)=2.32$, requires 2.45$]$. No other effect in the analysis approached conventional levels of significance. Note particularly the absence of any difference between eyes under any conditions.

The data appear to be roughly symmetrical about a low negative score rather than about zero. This downward drift from pre- to posttest is unexplained. It appears not to be produced by prism wearing, as, in the ND 0 condition, virtually identical scores are obtained for both base directions. Also, the hatched triangles at $\mathrm{ND} \infty$ represent monocular exposure to a restricted normal field. The true baseline for assessment of aftereffects should therefore be taken to be below zero.

Separate analyses were conducted for data collected under each of the two filter arrangements. With the filter on the "normal" eye it is clearly seen, from Fig. 1, that substantial aftereffects in the expected direction are obtained with monocular exposure (ND $\infty$ ) but that the magnitude decreases rapidly as the "normal" field becomes visible $[F$ base direction $(1,60)=15.13, p<.001 ; F$ Direction by Density $(4,60)=4.82, p<.01]$. With the filter on the prism eye, neither term approaches significance. Inspection of Fig. 1 suggests, however, a tendency for the functions to separate at medium to high filter densities. Such a trend would have considerable theoretical importance if it could be substantiated (cf. Foley, 1970), and for this reason, this part of the experiment was completely replicated by another $\mathrm{E}$, i.e., another $80 \mathrm{Ss}$ were run with five levels of density, two base directions, and two prism arrangements, but with the filter worn on the prism eye. Neither the base direction nor the Direction by Density terms were significant. An analysis was performed on the combined data from the two studies and the replications were shown to be not significantly different. Plotting the combined means tends to flatten the functions drawn in Fig. 1 and generally to bring them closer together, particularly in the critical area at ND 1.9. The overall negative bias occurs with comparable magnitude in the replication. Once again, there are no differences between eyes consistent with the differential exposure conditions.

\section{DISCUSSION}

The results of this study encourage the view that "normalcy" has particular potency in determining spatial judgments. As long as the luminance of the "normal" field is equal to or greater than that of the rearranged field, no aftereffect is measurable in either eye after a 10-min exposure. In fact, the "normal" field has to be dimmed by a factor in excess of 20 before reliable aftereffects can be measured. Even when the normal field is at little more than $1 / 100$ th the luminance of the prism field, the aftereffect is depressed. This is so regardless of the orientation of the accompanying prism. Therefore, the supremacy of the "normal" input cannot be attributed to the content of the visual field. (When the prism base is down, relatively more of the floor is included in the rearranged field.)

These experimental conditions, of course, do not themselves elucidate what "normal" vision means, e.g., whether the preference is built in or develops because of a long history of exposure. They do suggest that the system has a means of identifying normal input when it is accompanied by spatially rearranged information, and that its presence suppresses the processing of information in the rearranged field. It is important, however, to establish whether this comprises a true identification of the spatial norm as distinct from a preference mediated by contour-detection processes. The latter possibility arises because color fringes appear at regions of high contrast in the field of the prism, while they do not when the scene is viewed through plane glass. Contour breakdown through chromatic aberration could be an equivalent manipulation to the breakdown produced by reduced luminance. Alternatively, the presence or absence of color fringes could be regarded as a "give-away" clue to normalcy.

To test this possibility, we have twice conducted experiments where the ND 0 condition of the present study is compared with the identical condition except insofar as narrow-band green filters are worn on both eyes. In both experiments, eight groups of Ss have been run, one under each combination of two filter conditions (green filter present or absent), base direction (up or down), and prism arrangement (right or left eye). In one case, there were six Ss per group who received three successive 5-min exposures. In the other, eight Ss per group were exposed for $10 \mathrm{~min}$. In neither case did removal of the color fringe clue result in the emergence of aftereffects. On these grounds, we conclude that normal spatial information is recognizable and inhibits the acceptance of counter-information that is concurrently available.

\section{REFERENCES}

FOLEY, J. E., \& MIY ANISHI, K. Interocular effects in prism adaptation. Science, 1969, 165,311 .

FOLEY, J. E. Prism adaptation with opposed base orientations: The weighting of directional information from the two eyes. Perception \& Psychophysics, 8, 23-25.

HAJOS, A., \& RITTER, M. Interocular effects of 
adaptation to prismatic spectacles. Acta Psychologica, 1965, 24, 81-90.

HELD, R. Plasticity in sensory-motor systems Scientific American, 1965, $213,84-94$.

$$
\text { NOTES }
$$

1. This study was supported by the National
Research Council of Canada, Grant No. APA-1. University of Toronto. Toronto, Ontario, The assistance of Ariane Kudelska and $M$. Canada.

von Grunau in data collection and analysis was invaluable.

2. Address: Department of Psychology, (Accepted for publication February 16, 1970.) 